\title{
ESTABILIDADE DA COR DE DOCES EM MASSA DE POLPA DE UMBU (Spondias Tuberosa Arr. Cam.) NO ESTÁDIO DE MATURAÇÃO VERDE
}

\author{
Color stability of marmalade made from pulp of unripe umbu
}

\author{
Vânia Madeira Nunes Policarpo ${ }^{1}$, Soraia Vilela Borges², Érika Endo³, \\ Fernanda Travassos de Castro ${ }^{3}$, Aline Azevedo Damico ${ }^{4}$, Nilton Brito Cavalcanti ${ }^{5}$
}

\begin{abstract}
RESUMO
Os frutos de umbu (Spondias tuberosa Arr. Cam.) maduros são altamente perecíveis, e o aproveitamento deles no estádio de maturação verde, sob a forma de doce é uma interessante alternativa econômica, para o semi-árido nordestino. A conservação da cor, durante o armazenamento, constitui um fator de qualidade dos mais importantes para o consumo, e o presente trabalho objetivou avaliar sua alteração em função de diferentes formulações, embalagens (polipropileno e celofane) e temperaturas $\left(33\right.$ e $\left.43^{\circ} \mathrm{C}\right)$, por 90 dias de armazenamento. A adição de pectina e/ou xarope de glicose não alterou a luminosidade ( $\left.\mathrm{L}^{*}\right)$, mas diminuiu os valores de a* ( intensidade do vermelho ) e $\mathrm{b}^{*}$. ( intensidade do amarelo). Verificou-se diminuição dos valores de $\mathrm{L}^{*} \mathrm{a}^{*} \mathrm{e} \mathrm{b}^{*}$, notadamente para os doces armazenados à temperatura de $43^{\circ} \mathrm{C}$ e para a formulação sem adição de pectina. A embalagem não exerceu efeito significativo sobre $\mathrm{L}^{*}$ ( luminosidade), mas afetou $\mathrm{a}^{*} \mathrm{e} \mathrm{b}^{*}$ aos 90 dias de estocagem, em ambas as temperaturas. Os resultados globais indicaram o uso de pectina e xarope de glicose, embalagens de polipropileno e estocagem à temperaturas mais baixas como sendo as condições mais adequadas para preservação da cor.
\end{abstract}

Termos para indexação: Umbu (Spondias tuberosa Arr. Cam.), parâmetros de cor, vida de prateleira.

\section{ABSTRACT}

Ripe umbu (Spondias tuberosa Arr. Cam.) is highly perishable, thus using unripe umbu to make marmalade is an interesting economic alternative for the semi arid northeastern region in Brazil. Color conservation during storage is one of the major quality factors for consumption. This work aimed to evaluate color alteration in function of different formulations, packagings (polypropylene and cellophane) and temperatures $\left(33\right.$ and $\left.43^{\circ} \mathrm{C}\right)$ over 90 storage days. Pectin and/or glucose syrup adittion did not alter luminosity $\left(\mathrm{L}^{*}\right)$, but decreased $\mathrm{a}^{*}$ (red intensity) and $\mathrm{b}^{*}$ (yellow intensity) values. $\mathrm{L}^{*}$ (luminosity), $\mathrm{a}^{*}$ ( red intensity) and $\mathrm{b}^{*}$ (yellow intensity) values were found to decrease, especially for marmalade stored at $43^{\circ} \mathrm{C}$ and formulation without pectin addition. The package did not influenced the $\mathrm{L}^{*}$ (luminosity), but at 90 days of storage, this factor influenced the values of $\mathrm{a}^{*}$ and $\mathrm{b}^{*}$ parameters em both temperatures. The overall results indicated the use pectin-and glucose syrup-added formulation , packaging in polypropylene pots and storage at lower temperatures as being the most adequate conditions for color preservation.

Index terms: Umbu (Spondias tuberosa Arr. Cam.), color parameters, shelf-life.

(Recebido em 7 de julho de 2006 e aprovado em 20 de março de 2007)

\section{INTRODUCÃO}

O umbu é um fruto nativo da região semi-árida nordestina, considerado um produto vegetal de extração, e em 2003, foram coletadas no país, 9.132 toneladas de umbu, sendo que a participação da produção baiana, no total nacional foi de $86 \%$ (IBGE, 2005). Na época da safra, (dezembro a marco), há fartura de umbu maduro, que, no entanto, se deteriora em dois a três dias. As altas produções constituem uma fonte de renda para muitas famílias que, na época da safra, promovem a colheita dos frutos e os vendem para consumo "in natura", ou sob forma de doces, e têm contribuído para estabilização do êxodo rural do semiárido da Bahia. (CAVALCANTI et al., 2000).
O doce em massa de umbu é uma atividade artesanal de grande tradição no nordeste, que tem sido aplicada a polpas, em diferentes estádios de maturação (CAVALCANTI et al., 2000; POLICARPO et al., 2003; XAVIER, 1999), a fim de minimizar as perdas pós-colheita.

A cor é um dos principais atributos que afetam o consumo através da percepção da qualidade do produto, podendo até ser usada como estimativa dos componentes nutricionais para índices de qualidade, influenciando também na tomada de decisão de compra, pelo consumidor ((FRANCIS, 1995). A cor de doces em massa se deve aos pigmentos presentes nos alimentos e seus produtos de degradação. Há produtos derivados de reações enzimáticas e não enzimáticas (reação de Maillard, oxidação de

${ }^{1}$ Mestre - Departamento de Economia Doméstica-Instituto de Ciências Humanas-DED/ICHS - Universidade Federal Rural do Rio de Janeiro/UFRRJ Km 47 da Antiga Rodovia Rio-SP - 23851-970 - Seropédica, RJ - vaniapolicarpo@uol.com.br

2Doutor - Departamento de Ciência dos Alimentos/DCA - Universidade Federal de Lavras/UFLA - Cx. P. 3037 - $37200-000$ - Lavras, MG - sborges@ufla.br ${ }^{3}$ Mestres - Departamento de Tecnologia de Alimentos-DTA/TT-Instituto de Ciências Humanas - DED/ICHS - Universidade Federal Rural do Rio de Janeiro/UFRRJ Km 47 da Antiga Rodovia Rio-SP - 23851-970 - Seropédica, RJ -

${ }^{4}$ Mestre - Colorimetria - Serviço Nacional de Aprendizagem Industrial/SENAI - Rua Magalhães Castro, 174-20961-020-Riachuelo, RJ-adamico@cetigt.senai.b ${ }^{5}$ Mestre - Centro de Pesquisa Agropecuária do Trópico Semi-Árido/CPATSA - EMBRAPA - Br 128, km 152, Zona Rural - 56300-970 - Petrolina, PE nbrito@cpatsa.embrapa.br 
vitaminas), que são influenciados pelas condições de processamento (temperatura, tempo de cozimento), formulação (aditivos, concentração de açúcares, pH, pectina, dentre outros), (ALBUQUERQUE, 1997; DERVISI et al., 2001; JAWAHEER et al., 2003; WESCHE-EBELING et al., 1996; WICKLUND et al., 2005). Durante a estocagem, a estabilidade da cor é dependente da temperatura, material de embalagem (permeabilidade à luz, vapor de água e oxigênio), pH, dentre outros fatores (CARDOSO et al., 1997; FENNEMA ,1992; GARCIA-VIGUERA et al., 1999; WESCHE-EBELING et al., 1996; WICKLUND et al., 2005).

No umbu, os principais pigmentos são a clorofila e carotenóides conferindo-lhe uma cor verde-amarelada (estágio maduro), ou verde (estágio imaturo), além de pequena concentração e compostos fenólicos, os quais sofrem degradação devido aos motivos previamente citados, dando origem a pigmentos escuros. Este escurecimento se acentua durante o armazenamento destes produtos, a temperaturas mais elevadas (XAVIER, 1999). A clorofila é degradada por ácidos ou via enzimática em pigmentos verde-marron (feofitina ou feoforbida), (FENNEMA, 1992). Os carotenóides, quando processados, se tornam mais vulneráveis às alterações provocadas pelo calor, luz, concentração de oxigênio, durante o armazenamento, sofrendo auto-oxidação, originando compostos que escurecem o produto (FENNEMA, 1992; LIN \& CHEN, 2005; RAMESH et al., 2001).

Doces em massa de umbu são caracterizados por acidez elevada e por isto sofrem sinérese, durante o armazenamento (JACKIX, 1988; POLICARPO et al., 2003), caso não seja corrigido o $\mathrm{pH}$ e adicionado agentes, que tenham capacidade de reter esta umidade. São encontrados no comércio acondicionados, principalmente, em embalagens de celofane e potes de poliproprileno. A embalagem de celofane é mais econômica, mas é mais permeável à água do que as poliolefinas como o polipropileno (MORILLON et al., 2000); e à luz ( 79 a 90\% a 800 nm (LU et al., 2004), do que potes de polipropileno $(70 \%$ a $800 \mathrm{~nm}$ ) (ALVES et al., 2007), não sendo talvez tão apropriada para acondicionamento de doces em massa. Entretanto, não se encontra na literatura, nenhum estudo sobre o desempenho comparativo destas embalagens de doces em massa e particularmente, para doces em massa de umbu, neste estádio de maturação. Portanto, o objetivo desta pesquisa é verificar a influência da formulação, embalagem e tempo de armazenamento sobre a cor deste produto, a fim de selecionar as melhores condições de processamento e embalagem para o mesmo.

\section{MATERIAL E MÉTODOS}

Os frutos congelados, transportados via aérea (EMBRAPA-CPATSA), foram descongelados, lavados e sanitizados com solução 200 ppm de cloro, branqueados a $100^{\circ} \mathrm{C} / 5$ minutos, despolpados em despolpadeira Bertizzi (BERTIZZI, Rio de Janeiro) sendo a polpa acondicionada em sacos de polietileno, e armazenada sob congelamento, para posterior utilização. O processamento de obtenção do doce foi feito de acordo com o fluxograma descrito por Policarpo et al. (2003). Nesse processo experimental foram utilizadas três formulações, conforme Tabela 1, mantendo relação polpa/ açúcar de 1:1; o pH da polpa 3,2, corrigido com $\mathrm{CaCO}_{3}$. A pectina foi adicionada ao final do processo para não sofrer degradação devido à alta temperatura, durante o cozimento. Após o término do cozimento, os doces foram colocados, em quantidades constantes $(50 \mathrm{~g})$ em duas embalagens redondas: potes de polipropileno transparente (6 $\mathrm{cm}$ de diâmetro) e celofane (moldado em potes de polipropileno de igual dimensão). Eles foram armazenados nas temperaturas constantes de $33^{\circ} \mathrm{e} 43^{\circ} \mathrm{C}$ (estufa com temperatura controlada), e analisados em intervalos de tempo pré-determinados $(0,45$ e 90 dias). Os parâmetros de cor : L* (luminosidade), a* (intensidade de vermelho e verde ) e $\mathrm{b}^{*}$ (intensidade de amarelo e azul), foram medidos em um espectrofotômetro Minolta (modelo CM-3600D, Konica Minolta, Ramsey, NJ),

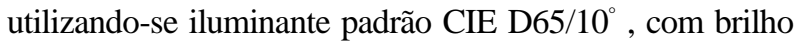
incluído, na temperatura de $25^{\circ} \mathrm{C}$, sendo os resultados expressos como a média de 8 determinações. O processo foi repetido 2 vezes, e os resultados foram tratados pelo teste de Tukey, a 5\% de probabilidade (PIMENTEL-GOMES, 1990).

TABELA 1 - Formulações de doces em massa.

\begin{tabular}{cccc}
\hline & $\begin{array}{c}\text { Xarope de } \\
\text { glicose } \\
(\boldsymbol{\%})\end{array}$ & $\begin{array}{c}\text { Pectina } \\
(\mathbf{g} / \mathbf{1 0 0} \mathbf{g})\end{array}$ & $\begin{array}{c}\text { Sólidos } \\
\text { solúveis } \\
\left({ }^{\circ} \text { Brix }\right)\end{array}$ \\
\hline F1 & 0 & 0,0 & 78 \\
F2 & 0 & 0,5 & 73 \\
F3 & 10 & 0,5 & 73 \\
\hline
\end{tabular}

\section{RESULTADOS E DISCUSSÃO}

As Tabelas 2 e 3 mostram a variação do parâmetro $\mathrm{L}^{*}$ (luminosidade), para as temperaturas de 33 e $43^{\circ} \mathrm{C}$, respectivamente, em função das formulações, embalagens e tempo de armazenamento. Não houve diferenças significativas para os fatores embalagem e formulação, uma vez que as duas embalagens são transparentes, embora a \% a transmissão de luz seja diferente para elas, conforme 
reportado na literatura (ALVES et al., 2007; LU et al., 2004). Também os níveis de aditivos adicionados não foram suficientes para promover diferenças, neste parâmetro. Embora não significativo o efeito dos aditivos, nota-se um ligeiro aumento na luminosidade, com o acréscimo do xarope de glicose, o qual é utilizado para aumentar o brilho destes produtos (JACKIX, 1988). Os valores da luminosidade tenderam a diminuir com o tempo de armazenamento em quaisquer temperatura, sendo registrados valores menores para $45^{\circ} \mathrm{C}$, e mais acentuadamente até 45 dias, a partir do qual esta degradação foi lenta. Ela se deve à oxidação de pigmentos presentes (clorofila, caroteno, compostos fenólicos ), os quais foram avaliados por Xavier (1999), em função da temperatura e presença/ausência de luz, mostrando que a temperatura foi o fator mais significativo na sua redução. Resultados similares foram obtidos por Garcia-Viguera et al. (1999), e Wicklund et al. (2005), avaliando a degradação de pigmentos antociânicos e mudança da luminosidade (instrumental), em geléias de morango, em várias temperaturas de estocagem.

Em relação às formulações, aos 90 dias de armazenamento em ambas as temperaturas, nota-se um menor percentual de perda para a formulação acrescida de pectina (F2), embalada em polipropileno $\left(13,97 \%\right.$ a $33^{\circ} \mathrm{C}$ e $19,6 \%$ a $\left.43^{\circ} \mathrm{C}\right)$. Similarmente, nota-se maiores perdas para a formulação acrescida de pectina e xarope de glicose (F3), em embalagens de polipropileno $\left(20,5 \%\right.$ a $33^{\circ} \mathrm{C}$ e $28 \%$ a $\left.43^{\circ} \mathrm{C}\right)$.

As Tabelas 4, 5, 6 e 7 mostram a variação do parâmetro a*, que varia de verde a vermelho, e $b^{*}$, que varia do amarelo para azul, para as temperaturas de 33 e $43^{\circ}$ $\mathrm{C}$, respectivamente, em função das formulações, embalagens e tempo de armazenamento. Observa-se que a adição de pectina e pectina/xarope de glicose alteraram os parâmetros $a^{*}$ e b* das formulações, obtendo-se uma formulação com tendência maior para vermelho e menor para amarelo, diminuindo a cor amarelo-esverdada, característica da polpa original. Tem sido reportado, em alguns trabalhos na literatura, que a adição de pectina (de baixo ou alto teor de metoxilação) diminui a intensidade de cor de geléias (DERVISI et al., 2001; NACHTIGALL et al., 2004; VENDRAMEL et al., 1997). Dervisi et al. (2001), testando crescentes níveis de pectina em geléias de morango, observaram decréscimo nos pigmentos antociânicos de geléia de morango e diminuição da intensidade da cor, sugerindo possível reação destes pigmentos com a pectina.

TABELA 2 - Efeito do tempo de armazenamento sobre o $L^{*}$ (luminosidade), no doce em massa de umbu à temperatura $33^{\circ} \mathrm{C}$, em diferentes períodos de armazenamento.

\begin{tabular}{|c|c|c|c|c|c|c|}
\hline \multirow[b]{3}{*}{$\mathbf{F}$} & \multicolumn{4}{|c|}{ Dias de armazenamento } & \multirow{2}{*}{\multicolumn{2}{|c|}{90}} \\
\hline & \multicolumn{2}{|c|}{$\mathbf{0}$} & \multicolumn{2}{|c|}{45} & & \\
\hline & EC & EP & EC & EP & EC & EP \\
\hline 1 & $32,19 \mathrm{aA}$ & $32,19 \mathrm{aA}$ & $26,55 \mathrm{aC}$ & $26,94 \mathrm{aB}$ & $27,99 \mathrm{aB}$ & $26,83 \mathrm{aB}$ \\
\hline 2 & $32,01 \mathrm{aA}$ & $32,01 \mathrm{aA}$ & $27,77 \mathrm{aB}$ & $28,99 \mathrm{aB}$ & $27,28 \mathrm{aB}$ & $27,56 \mathrm{aC}$ \\
\hline 3 & $35,36 \mathrm{aA}$ & $35,36 \mathrm{aA}$ & $28,61 \mathrm{aAB}$ & $28,61 \mathrm{aAB}$ & $27,70 \mathrm{aB}$ & $28,27 \mathrm{aB}$ \\
\hline
\end{tabular}

Médias seguidas da mesma letra minúscula na mesma coluna e mesma letra maiúscula na mesma linha, não diferem, estatisticamente, pelo teste de Tukey a 5\%.

EC: Embalagem de Celofane EP: Embalagem de Polipropileno.

TABELA 3 - Efeito do tempo de armazenamento sobre o $\mathrm{L}^{*}$ (luminosidade), no doce em massa de umbu à temperatura $43^{\circ} \mathrm{C}$, em diferentes períodos de armazenamento.

\begin{tabular}{|c|c|c|c|c|c|c|}
\hline & \multicolumn{4}{|c|}{ Dias de armazenamento } & \multirow{2}{*}{\multicolumn{2}{|c|}{90}} \\
\hline & \multicolumn{2}{|c|}{ 0 } & \multicolumn{2}{|c|}{45} & & \\
\hline & EC & EP & EC & EP & EC & EP \\
\hline $\mathrm{F} 1$ & $32,19 \mathrm{aA}$ & $32,19 \mathrm{aA}$ & $26,39 \mathrm{aC}$ & $26,61 \mathrm{aB}$ & $27,32 \mathrm{aB}$ & $26,07 \mathrm{aB}$ \\
\hline $\mathrm{F} 2$ & $32,01 \mathrm{aA}$ & $32,01 \mathrm{aA}$ & $27,08 \mathrm{aB}$ & $26,18 \mathrm{aB}$ & $25,48 \mathrm{aC}$ & $25,72 \mathrm{aC}$ \\
\hline F3 & $35,36 \mathrm{aA}$ & $35,36 \mathrm{aA}$ & $28,91 \mathrm{aAB}$ & $26,65 \mathrm{aB}$ & $26,20 \mathrm{aC}$ & $25,45 \mathrm{aC}$ \\
\hline
\end{tabular}

Médias seguidas da mesma letra minúscula na mesma coluna e mesma letra maiúscula na mesma linha, não diferem estatisticamente pelo teste de Tukey a $5 \%$.

EC: Embalagem de Celofane EP: Embalagem de Polipropileno. 
TABELA 4 - Efeito do tempo de armazenamento sobre o a* (intensidade de vermelho e verde), no doce em massa de umbu à temperatura de $33^{\circ} \mathrm{C}$, em diferentes períodos de armazenamento.

\begin{tabular}{clccccc}
\hline & \multicolumn{9}{c}{ Dias de armazenamento } & \multicolumn{2}{c}{$\mathbf{9 0}$} \\
& EC & EP & EC & EP & EC & EP \\
\hline F1 & $2,59 \mathrm{cB}$ & $2,59 \mathrm{cA}$ & $1,42 \mathrm{cC}$ & $1,40 \mathrm{cC}$ & $1,75 \mathrm{cB}$ & $3,06 \mathrm{aA}$ \\
$\mathrm{F} 2$ & $3,14 \mathrm{aA}$ & $3,14 \mathrm{aA}$ & $1,72 \mathrm{bC}$ & $1,73 \mathrm{bC}$ & $2,43 \mathrm{bB}$ & $2,05 \mathrm{cB}$ \\
$\mathrm{F} 3$ & $2,99 \mathrm{bA}$ & $2,99 \mathrm{bA}$ & $1,84 \mathrm{aC}$ & $1,84 \mathrm{aC}$ & $2,48 \mathrm{aB}$ & $2,35 \mathrm{bB}$ \\
\hline
\end{tabular}

Médias seguidas da mesma letra minúscula na mesma coluna e mesma letra maiúscula na mesma linha, não diferem estatisticamente pelo teste de Tukey a $5 \%$.

EC: Embalagem de Celofane EP: Embalagem de Polipropileno.

TABELA 5 - Efeito do tempo de armazenamento sobre o a* (intensidade de vermelho e verde) no doce em massa de umbu à temperatura de $43^{\circ} \mathrm{C}$, em diferentes períodos de armazenamento.

\begin{tabular}{ccccccc}
\hline \multicolumn{9}{c}{ Dias de armazenamento } \\
& EC & EP & EC & EP & EC & EP \\
\hline F1 & $2,59 \mathrm{cA}$ & $2,59 \mathrm{aA}$ & $1,00 \mathrm{aC}$ & $1,70 \mathrm{a} \mathrm{AB}$ & $1,40 \mathrm{aB}$ & $0,96 \mathrm{aC}$ \\
$\mathrm{F} 2$ & $3,14 \mathrm{a} \mathrm{A}$ & $3,14 \mathrm{aA}$ & $1,41 \mathrm{aAB}$ & $1,10 \mathrm{aC}$ & $0,79 \mathrm{aC}$ & $1,23 \mathrm{aB}$ \\
$\mathrm{F} 3$ & $2,99 \mathrm{~b} \mathrm{~A}$ & $2,99 \mathrm{aA}$ & $2,90 \mathrm{a} \mathrm{A}$ & $1,55 \mathrm{aB}$ & $0,80 \mathrm{aB}$ & $1,01 \mathrm{aC}$ \\
\hline
\end{tabular}

Médias seguidas da mesma letra minúscula na mesma coluna e mesma letra maiúscula na mesma linha, não diferem estatisticamente pelo teste de Tukey a $5 \%$.

EC: Embalagem de Celofane EP: Embalagem de Polipropileno.

TABELA 6 - Efeito do tempo de armazenamento sobre b* (intensidade de amarelo e azul) no doce em massa de umbu à temperatura de $33^{\circ} \mathrm{C}$, em diferentes períodos de armazenamento.

\begin{tabular}{ccccccc}
\hline & \multicolumn{9}{c}{ Dias de armazenamento } & \multicolumn{2}{c}{$\mathbf{9 0}$} \\
& EC & EP & EC & EP & EC & EP \\
\hline F1 & $9,29 \mathrm{aA}$ & $9,29 \mathrm{aA}$ & $2,88 \mathrm{cC}$ & $2,90 \mathrm{cC}$ & $4,91 \mathrm{aB}$ & $3,20 \mathrm{cB}$ \\
$\mathrm{F} 2$ & $8,63 \mathrm{cA}$ & $8,63 \mathrm{bA}$ & $3,51 \mathrm{bC}$ & $3,50 \mathrm{bC}$ & $3,81 \mathrm{cB}$ & $4,46 \mathrm{bB}$ \\
$\mathrm{F} 3$ & $9,29 \mathrm{aA}$ & $9,29 \mathrm{aA}$ & $4,65 \mathrm{aC}$ & $4,65 \mathrm{aC}$ & $4,80 \mathrm{bB}$ & $5,01 \mathrm{cB}$ \\
\hline
\end{tabular}

Médias seguidas da mesma letra minúscula na mesma coluna e mesma letra maiúscula na mesma linha, não diferem, estatisticamente, pelo teste de Tukey a 5\%.

EC: Embalagem de Celofane EP: Embalagem de Polipropileno.

TABELA 7 - Efeito do tempo de armazenamento sobre o $b^{*}$ (intensidade de amarelo e azul) no doce em massa de umbu à temperatura de $43^{\circ} \mathrm{C}$, em diferentes períodos de armazenamento .

\begin{tabular}{ccccccc}
\hline & \multicolumn{9}{c}{ Dias de armazenamento } & \multicolumn{2}{c}{$\mathbf{9 0}$} \\
& $\mathbf{E C}$ & $\mathbf{E P}$ & $\mathbf{E C}$ & $\mathbf{E P}$ & $\mathbf{E C}$ & $\mathbf{E P}$ \\
\hline 1 & $9,29 \mathrm{aA}$ & $9,29 \mathrm{aA}$ & $2,00 \mathrm{cC}$ & $2,40 \mathrm{bB}$ & $2,99 \mathrm{aB}$ & $1,97 \mathrm{bC}$ \\
2 & $8,63 \mathrm{bA}$ & $8,63 \mathrm{bA}$ & $2,70 \mathrm{bB}$ & $1,80 \mathrm{cC}$ & $1,56 \mathrm{bC}$ & $2,35 \mathrm{aB}$ \\
3 & $9,29 \mathrm{aA}$ & $9,29 \mathrm{aA}$ & $4,90 \mathrm{aB}$ & $2,50 \mathrm{aB}$ & $1,52 \mathrm{cC}$ & $1,69 \mathrm{cC}$ \\
\hline
\end{tabular}

Médias seguidas da mesma letra minúscula na mesma coluna e mesma letra maiúscula na mesma linha, não diferem, estatisticamente, pelo teste de Tukey a $5 \%$.

EC: Embalagem de Celofane EP: Embalagem de Polipropileno. 
Durante o armazenamento, houve uma tendência à diminuição progressiva de $\mathrm{a}^{*} \mathrm{e} \mathrm{b}^{*}$ principalmente à temperatura de $43^{\circ} \mathrm{C}$ e para a Formulação F1, indicando perda da cor verde-amarelo, característicos de polpas e doces recém-produzidos, devido à oxidação dos pigmentos, conforme já discutida em parágrafos anteriores. Tais resultados são semelhantes aos observados por Cardoso et al. (1997), os quais atribuíram à mudança de cor da geléia de jambo à degradação de antocianinas, intensificada principalmente pelo aumento da temperatura de armazenamento. Similares conclusões foram encontradas por vários autores na estocagem de geléias de diferentes frutas (GARCIA-VIGUERA et al., 1999; WHESCHEEBELING et al., 1995; WICKLUND et al., 2005) e estocagem de suco de maracujá (SANDI et al., 2003).

A embalagem exerceu influência significativa sobre os parâmetros $\mathrm{a}^{*}$, (intensidade do vermelho e verde) e b* (intensidade do vermelho a verde), somente aos 90 dias de armazenamento, em ambas as temperaturas de armazenamento. A $33^{\circ} \mathrm{C}$ para produtos embalados em potes de polipropileno, observou-se um aumento da intensidade do vermelho $\left(\mathrm{a}^{*}\right)$ para a $\mathrm{F} 1$, em relação ao embalado em celofane e o contrário foi verificado a $43^{\circ} \mathrm{C}$. Para as formulações F2 (pectina) e F3 (pectina mais xarope de glicose), os valores $\mathrm{a}^{*}$ atingidos $\mathrm{a}^{\circ} \mathrm{C}$, foram maiores do que os atingidos pela embalagem de celofane. Quanto aos valores de $\mathrm{b}^{*}$ (intensidade do amarelo), a $33^{\circ} \mathrm{C}$, nota-se maiores valores atingidos para as formulações F2 (pectina) e F3 (pectina mais xarope de glicose) em embalagens de polipropileno, e maiores valores para $\mathrm{F} 1$, em embalagens de celofane. A $43^{\circ} \mathrm{C}$, a mesma tendência similar foi observada. Estes resultados sugerem que a proteção contra uma maior alteração de cor, oferecida pela embalagem de polipropileno, seja pela sua baixa permeabilidade à água e menor permeabilidade à luz, uma vez que ambos os fatores desencadeiam reações químicas diversas nos alimentos, que culminam na alteração de cor, conforme já comentado previamente neste trabalho.

Em relação à degradação dos parâmetros a* (intensidade do vermelho e verde) e $b^{*}$ (intensidade do amarelo e azul), das diferentes formulações, observou-se, ao fim do armazenamento (90 dias), a maior variação registrada para $a^{*}$, (intensidade do vermelho a verde), foi para formulação acrescida de pectina $\left(34 \%\right.$ a $\left.33^{\circ} \mathrm{C}\right)$, embalada em potes de polipropileno. A menor variação de $a^{*}$ foi para formulação F1 embalada em celofane, (18\% a $33^{\circ} \mathrm{C}$ ). Para a formulação acrescida de xarope de glicose (F3), a perda foi de $21 \%$ a $33^{\circ} \mathrm{C}$. A $43^{\circ} \mathrm{C}$, o percentual de degradação do parâmetro $\mathrm{a}^{*}$, para todas as formulações, foi acima de $60 \%$. Quanto ao parâmetro b* (intensidade do amarelo-azul), observou-se menores perdas para F3 embalada em polipropileno $\left(46 \%\right.$ a $\left.33^{\circ} \mathrm{C}\right)$ e maiores perdas para $\mathrm{F} 1$ embalada em celofane $\left(65 \%\right.$ a $\left.33^{\circ} \mathrm{C}\right)$. A $43^{\circ} \mathrm{C}$, as perdas superam os $70 \%$, registrando menores perdas para F2 (pectina), em embalagens de polipropileno.

\section{CONCLUSÃO}

Do ponto de vista da conservação da cor amareloesverdeada do doce, sugere-se a adição de pectina e xarope de glicose, devido à obtenção de produto de cor mais clara, e embalagem de polipropileno, pois menores alterações foram observadas nos parâmetros de cor ( $a^{*}$ e b*), nestas embalagens em relação ao celofane, em temperaturas mais amenas $\left(33^{\circ} \mathrm{C}\right)$.

\section{REFERÊNCIAS BIBLIOGRÁFICAS}

ALBUQUERQUE, J. P. Fatores que influem no processamento de geléias e geleadas de frutas. Boletim da SBCTA, Campinas, v. 31, n. 1, p. 62-67, 1997.

ALVES, R. M.; DENDER, A. G. F. van; JAIME, S. B. M.; MORENO, I.; PEREIRA, B. C. Effect of light and packages on stability of spreadable processed cheese. International of Dairy Journal, Oxford, v. 17, p. 365-373, 2007.

CARDOSO, R. L.; FERREIRA, V. L. P.; MONTGOMERY, M. W.; YOTSUYANAGI, K. Efeito do tempo, luz e temperatura na cor da geléia de jambo vermelho (Eugeniamalaccensis, Lin). Ciência e Tecnologia de Alimentos, Campinas, v. 17, p. 28-31, 1997.

CAVALCANTI, N. B.; RESENDE, G. M.; BRITO, L. T. L. Processamento do fruto de umbuzeiro. Ciência e Agrotecnologia, Lavras, v. 24, n. 1, p. 252-259, 2000.

DERVISI, P.; LAMB, J.; ZABETAKIS, I. High pressure processing in jam manufacture: effects on textural and colour properties. Food Chemistry, London, v. 73, p. 85-91, 2001.

FENNEMA, O. R. Química de los alimentos. Zaragoza: Acríbia, 1992. 1095 p.

FRANCIS, F. J. Quality as influencied by color. Food Quality and Preference, Barking, v. 6, p. 149-155, 1995.

GARCIA-VIGUERA, C.; ZAFRILLA, P.; ROMERO, F.; ABELLÁN, P.; ARTÉS, F.; TOMÁS-BARBERÁN, F. A. Color stability of strawberry jam as affected ay cultivar and storage temperature. Journal of Food Science, Chicago, v. 64, n. 2, p. 243-247, 1999. 
INSTITUTO BRASILEIRO DE GEOGRAFIA E ESTATÍSTICA. Produção da extração vegetal e da sivilcultura: Brasil 2002-2003. Disponível em: <http:// www.ibge.gov.br>. Acesso em: 4 ago. 2005.

JACKIX, M. H. Doces, geléias e frutas em calda. São Paulo: Ícone, 1988. 254 p.

JAWAHEER, B.; GOBURDHUN, D.; RUGGOO, A. Effect of processing and storage of guava into jam and juice on the ascorbic acid content. Plant Foods for Human Nutrition, Dordrecht, v. 58, p. 1-12, 2003.

LIN, C. H.; CHEN, B. H. Stability of carotenoids in tomato juice during storage. Food Chemistry, London, v. 90, p. 837-846, 2005.

LU, Y.; ZHANG, L.; XIAO, P. Structure, properties and biodegradability of water resistant regenerated cellulose films coated with polyurethane/benzyl konjac glucomannan semi-IPN coating. Polymer Degradation and Stability, Brighton, v. 86, p. 51-57, 2004.

MORILLON, V.; DEBEAUFORT, F.; BLOND, G.; VOILLER A. Temperature influence on moisture transfer through synthetic films. Journal of Membrane Science, Atlanta, v. 168, p. 223-231, 2000.

NACHTIGALL, A. M.; SOUZA, E. L.; MALGARIM, M. B.; ZAMBIAZI, R. C. Geléias light de amora-preta. Boletim do Centro de Pesquisa e Processamento de Alimentos, Curitiba, v. 22, n. 2, p. 337-353, 2004.

PIMENTEL-GOMES, F. Curso de estatística experimental. 13. ed. Piracicaba: Nobel, 1990. 467 p.

POLICARPO, V. M. N.; RESENDE, J.; ENDO, E.; MARCUSSI, B.; CASTRO, F. T.; JORGE, E. C.; BORGES, S. V.; CAVALCANTI, N. B. Aprovechamiento de la pulpa de umbu (Spondias tuberosa, Arr. Cam.) verde como alternativa para la producción dulces em masa. Alimentaria, Madrid, n. 344, p. 75-78, 2003.

RAMESH, M. N.; WOLF, W.; TEVINI, W.; JUNG, G. Influence of processing parameters on the drying of spice paprika. Journal of Food Engineering, Essex, v. 49, p. 6372, 2001.

SANDI, D.; CHAVES, J. B. P.; SOUZA, A. C. G.; SILVA, M. T. C.; PARREIRAS, F. M. Correlação entre características físico-químicas e sensoriais em suco de maracujá amarelo (Passiflora edulis var. flavicarpa) durante o armazenamento. Ciência e Tecnologia de Alimentos, Campinas, v. 23, n. 3, p. 355-361, 2003.

VENDRAMEL, S. M. R.; CÂNDIDO, L. M. B.; CAMPOS, A. M. Avaliação reológica e sensorial de geléias com baixo teor de sólidos solúveis com diferentes hidrocolóides obtidas a partir de formulações em pó. Boletim do Centro de Pesquisa e Processamento de Alimentos, Curitiba, v. 15, n. 1, p. 37-56, 1997.

WESCHE-EBELING, P.; ARGAIZ-JAMET, A.; HERNANDEZ-PORRAS, L. G.; LÓPEZ-MALO, A. Presevation factors and processing effects on anthocyanin pigments in plums. Food Chemistry, London, v. 57, n. 3, p. 399-403, 1996.

WICKLUND, T.; ROSENFELD, H. J.; MARTINSEN, B. K.; SUNDFOR, M. W.; LEA, P.; BRUUN, T.; BLOMHOFF, R.; HAFFNER, K. Antioxidant capacity and colour of strawberry jam as influenced by cultivar and storage conditions. Lebensmittel Wissenschaft und Technology, London, v. 38, p. 387-391, 2005.

XAVIER, A. N. Caracterização química e vida-de-prateleira do doce em massa de umbu. 1999. 82 f. Dissertação (Mestrado em Ciência e Tecnologia dos Alimentos) Universidade Federal de Minas Gerais, Belo Horizonte, 1999. 